\title{
Transcriptomic profiling of experimental arterial injury reveals new mechanisms and temporal dynamics in vascular healing response
}

\author{
Samuel Röhl, MD, PhD, ${ }^{a}$ Urszula Rykaczewska, MSc, ${ }^{a}$ Till Seime, MSc, ${ }^{a}$ Bianca E. Suur, MSc, ${ }^{a}$ \\ Maria Gonzalez Diez, PhD, ${ }^{b}$ Jesper R. Gådin, PhD, ${ }^{b}$ Anastasiia Gainullina, MSc, ${ }^{c}$ Alexey A. Sergushichev, PhD, \\ Robert Wirka, MD, PhD, ${ }^{d}$ Mariette Lengquist, MSc, ${ }^{a}$ Malin Kronqvist, MSc, ${ }^{a}$ Otto Bergman, $\mathrm{PhD},{ }^{b}$ \\ Jacob Odeberg, MD, PhD, Jan H. N. Lindeman, MD, PhD, ${ }^{e}$ Thomas Quertermous, MD, PhD, ${ }^{d}$ \\ Anders Hamsten, MD, PhD, ${ }^{b}$ Per Eriksson, MSc, PhD, ${ }^{b}$ Ulf Hedin, MD, PhD, ${ }^{a}$ Anton Razuvaev, MD, PhD, ${ }^{a}$ and \\ Ljubica Perisic Matic, MSc, PhD, ${ }^{a}$ Solna and Stockholm, Sweden; St. Petersburg, Russia; Stanford, Calif; and Leiden, \\ The Netherlands
}

\begin{abstract}
Objective: Endovascular interventions cause arterial injury and induce a healing response to restore vessel wall homeostasis. Complications of defective or excessive healing are common and result in increased morbidity and repeated interventions. Experimental models of intimal hyperplasia are vital for understanding the vascular healing mechanisms and resolving the clinical problems of restenosis, vein graft stenosis, and dialysis access failure. Our aim was to systematically investigate the transcriptional, histologic, and systemic reaction to vascular injury during a prolonged time.
\end{abstract}

Methods: Balloon injury of the left common carotid artery was performed in male rats. Animals $(n=69)$ were euthanized before or after injury, either directly or after 2 hours, 20 hours, 2 days, 5 days, 2 weeks, 6 weeks, and 12 weeks. Both injured and contralateral arteries were subjected to microarray profiling, followed by bioinformatic exploration, histologic characterization of the biopsy specimens, and plasma lipid analyses.

Results: Immune activation and coagulation were key mechanisms in the early response, followed by cytokine release, tissue remodeling, and smooth muscle cell modulation several days after injury, with reacquisition of contractile features in later phases. Novel pathways related to clonal expansion, inflammatory transformation, and chondro-osteogenic differentiation were identified and immunolocalized to neointimal smooth muscle cells. Analysis of uninjured arteries revealed a systemic component of the reaction after local injury, underlined by altered endothelial signaling, changes in overall tissue bioenergy metabolism, and plasma high-density lipoprotein levels.

Conclusions: We demonstrate that vascular injury induces dynamic transcriptional landscape and metabolic changes identifiable as early, intermediate, and late response phases, reaching homeostasis after several weeks. This study provides a temporal "roadmap" of vascular healing as a publicly available resource for the research community. (JVSVascular Science 2020;1:13-27.)

Clinical Relevance: Endovascular intervention causes an injury to the arterial wall that subsequently induces a healing response to restore the vessel wall homeostasis. Complications after vascular interventions related to defective or excessive healing response, such as thrombosis or restenosis, are common and result in increased morbidity, suffering of the patient, need for repeated interventions, and possibly death. Thus, there is a need for better understanding of the underlying molecular mechanisms during vascular injury and healing response to identify and to assess the risk of complications in patients. Using an experimental model of vascular injury, this study demonstrates the full landscape of dynamic transcriptional changes in the resolution of vascular injury, accompanied also by systemic variations in plasma lipid levels and reaching homeostasis several weeks after injury. These

\footnotetext{
From the Department of Molecular Medicine and Surgery, and Department of

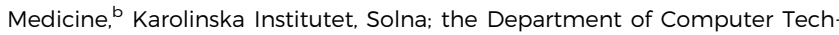
nologies, ITMO University, St. Petersburg'; the Department of Cardiovascular Medicine, Stanford University School of Medicine, Stanford ${ }^{d}$; the Department of Protein Science, School of Chemistry, Biotechnology and Health, Royal Institute of Technology, Science for Life Laboratory, Sweden, and the Department of Haematology, Coagulation Unit, Karolinska University Hospital, Stockholm ${ }^{\mathrm{e}}$; and the Leiden University, Leiden. ${ }^{f}$

This work was funded by the European Union's Horizon 2020/Marie Sklodowska-Curie grant agreement No. 722609 (INTRICARE); The Swedish Heart and Lung Foundation; Swedish Research Council; Stockholm County Council; and Swedish Society for Medical Research and Karolinska Institute Foundations.
}

Author conflict of interest: none.
Additional material for this article may be found online at www.jvascsurg.org. Correspondence: Ljubica Perisic Matic, MSc, PhD, Assistant Professor, Division of Vascular Surgery, Department of Molecular Medicine and Surgery, Karolinska Institutet, BioClinicum J8:20, Visionsgatan 4, 17164 Solna, Sweden (e-mail: ljubica.matic@ki.se).

The editors and reviewers of this article have no relevant financial relationships to disclose per the JVS-Vascular Science policy that requires reviewers to decline review of any manuscript for which they may have a conflict of interest. 2666-3503

Copyright $\odot 2020$ by the Society for Vascular Surgery. Published by Elsevier Inc This is an open access article under the CC BY-NC-ND license (http:// creativecommons.org/licenses/by-nc-nd/4.0/).

https://doi.org/10.1016/j.jvssci.2020.01.001 
results can guide the development of new strategies and molecular targets for modulation of the intimal response on endovascular interventions.

Keywords: Rat carotid artery balloon injury; Vessel wall healing; Gene expression microarrays; Smooth muscle cells

Endovascular intervention causes an injury to the arterial wall that subsequently induces a healing response to restore vessel wall homeostasis ${ }^{1,2}$ Complications after vascular interventions related to defective or excessive healing response, such as thrombosis or restenosis, are common and result in increased morbidity, suffering of the patient, repeated interventions, and possibly death. Detection and diagnosis of arterial disease currently rely on clinical evaluation and radiologic examination, and there is a need for better understanding of the underlying mechanisms to identify and to assess the risk of acute complications in patients.

The arterial wall healing response roughly develops through three major components: acute immune reaction after de-endothelialization, intimal hyperplasia formation, and vascular remodeling., ${ }^{3,4}$ On arterial injury, the endothelial lining is disrupted, which exposes the subendothelial matrix to the bloodstream, resulting in local thrombosis and recruitment of circulating leukocytes to the vessel wall. Re-endothelialization is crucial to reduce the risk of thrombosis and to restore vascular wall homeostasis. ${ }^{5}$ In response to disruption of the endothelial lining and traumatic mechanical stretch, medial vascular smooth muscle cells (SMCs) become activated and undergo a phenotypic switch from quiescent to dedifferentiated, synthetic SMCs with proliferative potential. Immune response and activation of matrix metalloproteinases promote detachment of the activated SMCs from the basement membrane and the surrounding extracellular matrix (ECM), which is followed by migration of cells to the intima. ${ }^{6}$ Intimal SMCs proliferate and secrete ECM components that form the neointima, ${ }^{7}$ and simultaneously, a vascular remodeling process is initiated that is characterized by ECM modulation, release of ECM-bound growth factors, and collagen deposition, resulting in arterial stiffening and changed lumen diameter. ${ }^{8}$

Activation of SMCs is accompanied by alterations in gene expression that result in downstream effects with changes in their morphologic and physiologic characteristics. ${ }^{9}$ The gene expression patterns during phenotypic switching of SMCs are complex and dynamic ${ }^{10-12}$; in addition, SMCs can display a high grade of plasticity in vascular diseases, assuming functional characteristics primarily related to other cell lineages, such as myeloid cells, macrophages, chondrocytes, and endothelial cells. ${ }^{9}$ Altogether, this complicates the accurate identification and tracking of SMC fate; hence, a detailed map of gene expression variations over time would be a powerful tool to further understand the diversity and function of these cells in the complex process of arterial healing.

The rat common carotid artery balloon injury is an established model for studies of arterial wall healing and intimal hyperplasia formation..$^{13-15}$ The model was originally developed by Clowes et $\mathrm{al}^{15}$ in the early $1980 \mathrm{~s}$ and has since been an important experimental platform for investigating arterial physiology in pharmacologic studies. More recently, availability of global transcriptomic profiling technologies has offered new opportunities to generate deeper molecular insights into the complex biologic processes, such as arterial wall healing. In addition, the concept of biobanking coupled with large-scale data sets and open resources is increasingly encouraged in human research and recognized to facilitate broader scientific collaborations, ${ }^{16,17}$ but it has not yet been widely applied in animal studies. Recognizing this gap, we employed the rat balloon injury model with a systematic biobanking approach across different time points (hours to months), combined with multilevel gene expression analyses by microarrays, histologic evaluation, and plasma metabolic measurements, to generate a novel resource for rat arterial wall healing (Biobank of Rat Carotid Artery [BiRCA]). Our integrative investigations from this animal biobank offer a comprehensive understanding of the natural course of arterial injury and healing response and reveal novel pathways and key molecular signatures implicated in this process while also confirming many others that were previously described in validation of this new resource provided for the broader community.

\section{METHODS}

Animals and study design. Male Sprague-Dawley rats (Charles River, Scanbur Research A/S, Sollentuna, Sweden) were housed at an animal facility in enriched cages supervised by professional animal caretakers. The animals ( $n=69$ ), with an average bodyweight of 300 to $400 \mathrm{~g}$, were randomized into nine groups ( $\mathrm{n}=7-10$ / group). As outlined in Supplementary Fig 1 (online only), eight groups were subjected to left common carotid artery balloon injury and euthanized at different time points after surgery (O hours, 2 hours, 20 hours, 2 days, 5 days, 15 days, 6 weeks, and 12 weeks); one group of seven animals was used as intact control. Analgesics (buprenorphine, $0.01 \mathrm{mg} / \mathrm{kg}$ [Temgesic; RB Pharmaceuticals Ltd, Berkshire, Great Britain) were administered when needed. On euthanization, both injured and 
uninjured (contralateral right common carotid arteries) tissue was harvested for transcriptomic and histologic analyses; other organs (heart, liver, aorta) and blood were collected for serum and plasma separation and plasma cholesterol measurements.

All animal experiments were approved by the Stockholm Ethical Board (Dnr N181/16; N137/14), and institutional guidelines for animal care were followed. A detailed description of all methods as well as of statistical and bioinformatic analyses is given in the Supplementary Methods (online only).

\section{RESULTS}

Histologic changes in response to vascular injury. Gross histologic changes in response to injury were assessed using Masson trichrome and Movat pentachrome stains (Supplementary Fig 2, online only). The earliest changes could be observed at 2 to 20 hours after injury with increasing cell mass in adventitia, which was most pronounced at 5 days. Although neointimal thickening did not become prominent until 2 weeks after injury, the first cells were observed in the intima 5 days after injury, whereas changes in the ECM composition were visible 2 hours after injury. In particular, increased proteoglycan staining appeared at 2 hours, initially confined to the luminal layer of the artery and later spread to the deeper layers, whereas collagen staining increased within the media and neointima from 2 weeks. Interestingly, at 12 weeks after injury, distinct elastin staining was observed in the luminal layer of the neointima, and neointimal cells assumed a more elongated shape.

This assessment confirmed and extended the previously described basic changes in response to vascular injury $^{4}$ and laid the ground for more detailed bioinformatic analyses.

Global transcriptomic profiling indicates three healing stages through acute response, tissue remodeling, and late resolution phase. First, overall gene expression profiles during the time course of vessel injury and healing were assessed by principal components analysis (PCA). In injured arteries (Fig 1, A), PCA showed that an early acute response could be distinguished after 2 hours compared with intact arteries. The intermediate remodeling phase at 20 hours, 2 days, and 5 days after injury showed clearly separate global profiles during the chronic healing response. Interestingly, late time points at 2 weeks, 6 weeks, and 12 weeks after injury clustered again near the intact arteries, indicating their overall closeness in expression patterns and suggesting resolution of healing and return to baseline tissue homeostasis. Of note, there was little variation within the clusters, indicating a homogeneous healing response, and no similar clustering was observed by PCA in uninjured arteries.

\section{ARTICLE HIGHLIGHTS}

Type of Research: Experimental animal research - Key Findings: Bioinformatic analyses of microarrays from the rat vascular injury model revealed immune activation and coagulation as key mechanisms in the early response phase, followed by tissue remodeling. cytokine activation, and smooth muscle cell migration several days after injury; reacquisition of contractile features occurred in late stages. Novel pathways related to clonal expansion, inflammatory transformation, and chondro-osteogenic differentiation were also found and localized to neointimal smooth muscle cells. The dynamic transcriptional changes were accompanied by systemic variations in plasma lipid levels, also reaching homeostasis several weeks after injury.

- Take Home Message: Vascular injury induces dynamic transcriptional landscape and metabolic changes identifiable as early, intermediate, and late response phases, reaching homeostasis after several weeks. This study provides a temporal "roadmap" of vascular healing as a resource available for the research community.

Differential gene expression was thereafter assessed by comparing microarray profiles at each time point against the previous one (Fig 1, B; Supplementary Tables I-VIII, online only). As visualized by volcano plots, repression of genes was more pronounced in early phases, with the biggest fold changes compared with other phases. As expected, endothelial markers (von Willebrand factor Podxl) were lost directly after injury, whereas interleukin (IL) 6 was strongly repressed at both 2 hours and 20 hours after injury. Immediate early induction (ie, of NK cell marker Cleclb and later of innate immunity-linked TIr7 and leukocyte chemokine $\mathrm{C} \times 3 \mathrm{crl}$ and Ednrb involved in vasoregulation) was also noted up to 20 hours after injury, when gene upregulation in general became more prominent. Fold changes in gene expression were smaller during the intermediate tissue remodeling stages and subsided even more during the late-stage response. At day 5 after injury, ECM markers such as tenascin N (Tnn), thrombospondin 4 (Thbs4), and Clq/tumor necrosis factor-related protein 3 (Clqtnf3) were the most upregulated. This trend was succeeded by the upregulation of actin cytoskeleton markers such as Actal at 2 weeks after injury but also corticotropinrelated (Crhr2) and thyroid hormone-related (Thrsp) genes and osteoblast marker (Bmp2) at late stages. Concomitantly, proliferation markers (Cdkn3) and matrix degradation (matrix metalloproteinase 12) were gradually downregulated.

Overall, this first level of analyses showed that the highest differential gene expression regulation appeared at 


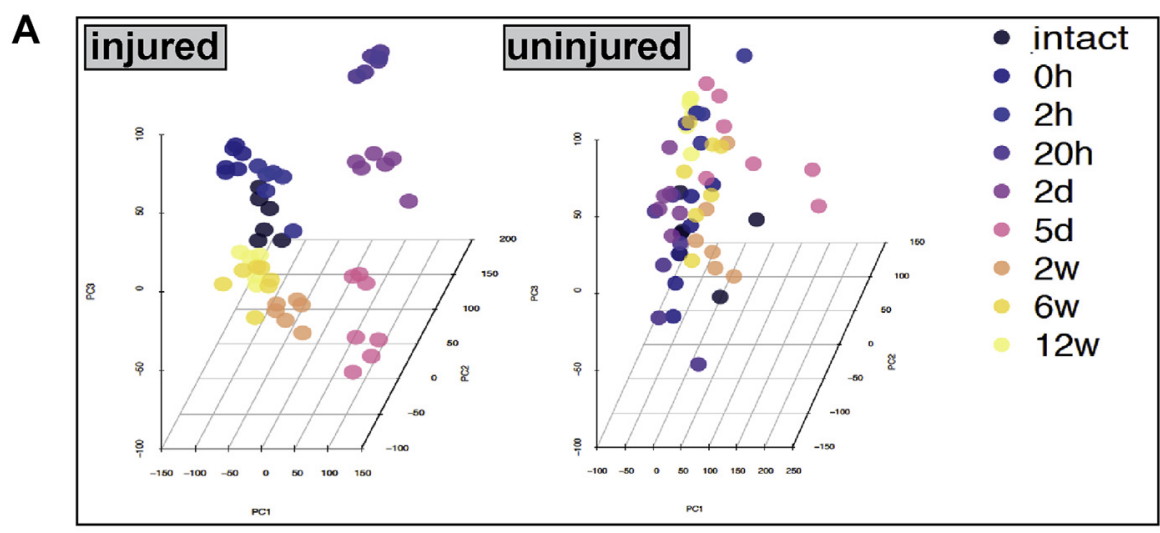

B
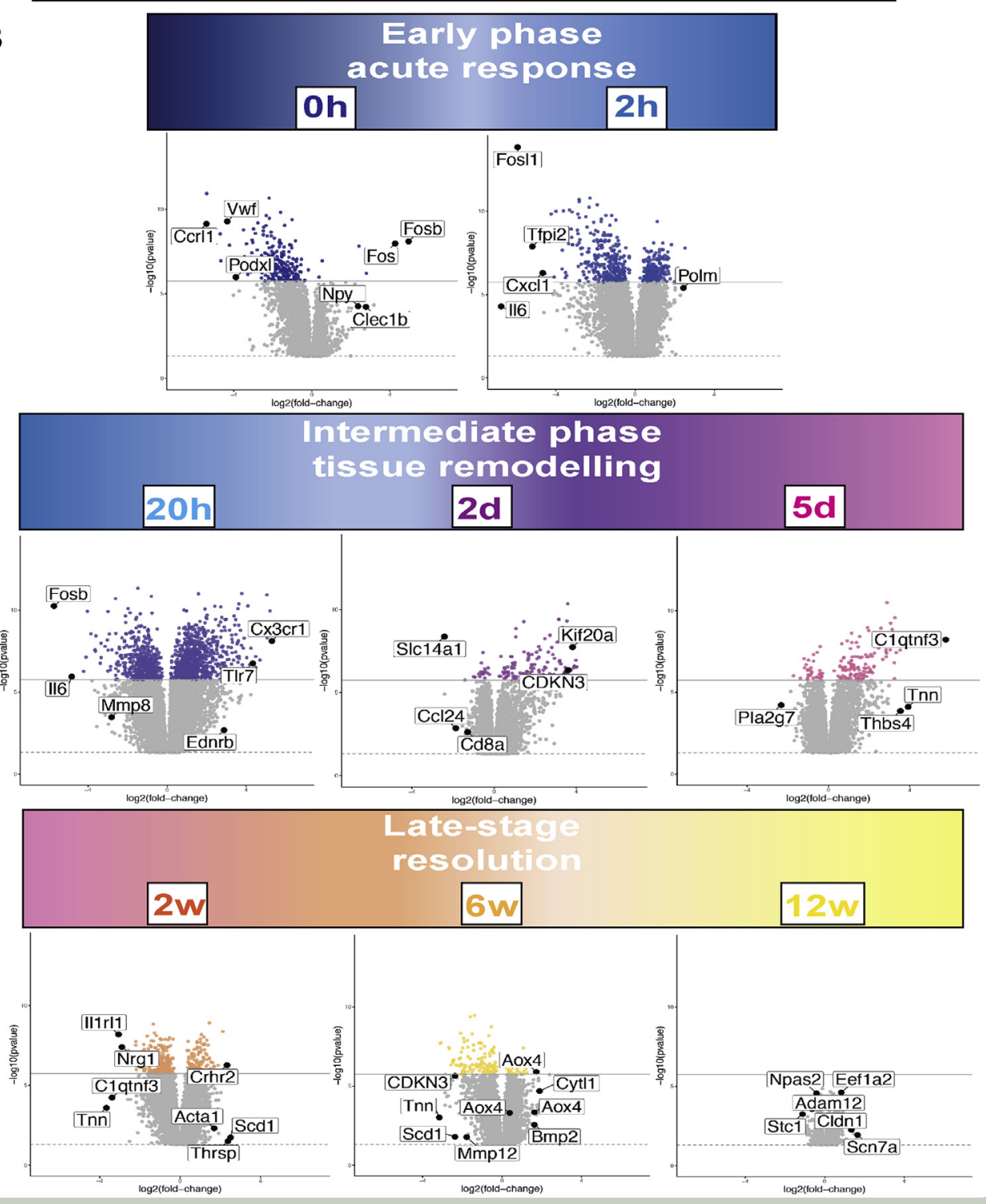

Fig 1. Global transcriptomic changes in response to vascular injury. A, Principal components analysis (PCA) of transcriptomic profiles from both injured and uninjured arteries, including intact vessels and vessels collected at 2 hours, 20 hours, 2 days, 5 days, 2 weeks, 6 weeks, and 12 weeks after injury. B, Differential gene expression changes during the time course of healing in injured arteries visualized by volcano plots. Microarray analysis was performed by comparing each time point against the previous one. Selected downregulated and upregulated genes are highlighted in the plots. The dashed line indicates nominal $P$ value threshold, and the solid line indicates $P$ values significant after Bonferroni correction. 


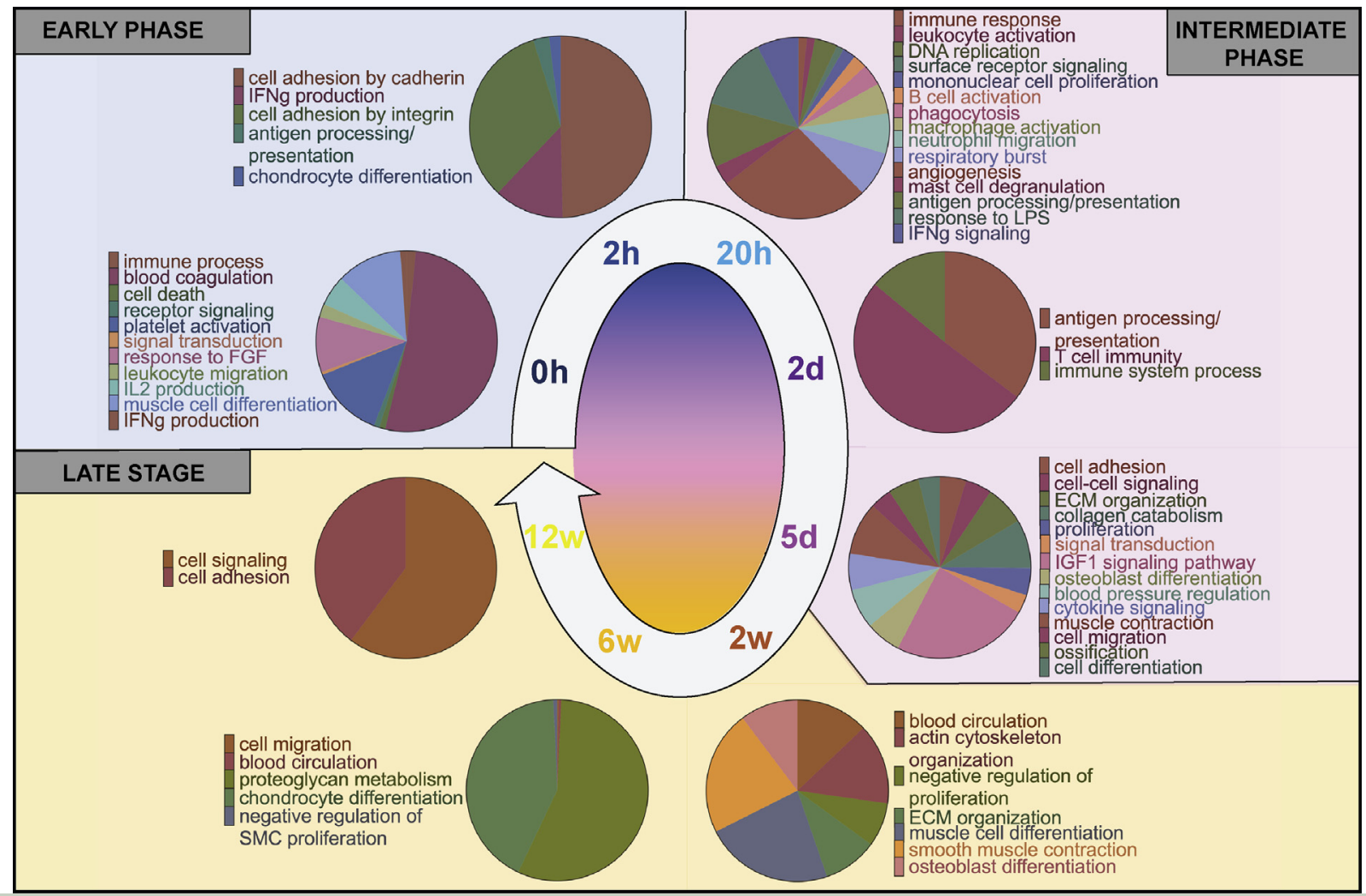

Fig 2. Bioinformatic pathway analyses characterize three major phases in response to injury. Gene set enrichment analyses of significantly upregulated genes at each time point on injury vs the previous one. Rapid induction of immune response and coagulation during early acute phase, followed by extracellular matrix (ECM) remodeling, muscle contraction, and ossification in intermediate phase, resolved by gradual attenuation of smooth muscle cell (SMC) proliferation in late phase, as observed in injured arteries. Plots represent enrichment of gene ontology categories; only significant processes with $P$ value $<.05$ are shown. FGF, Fibroblast growth

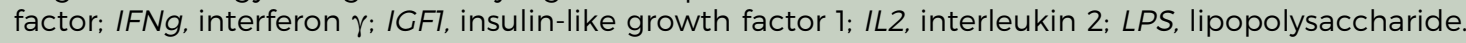

early time points, subsiding in the intermediate healing stages and reaching homeostasis in late resolution phase several weeks after injury.

Gene set enrichment analyses reveal key pathways, molecular signatures, and subcellular compartments driving vessel wall healing. Enrichment analyses of gene ontology processes based on the significantly upregulated genes (Fig 2) in injured arteries showed early response associated mostly with coagulation, inflammation, and interferon $\gamma($ IFN- $\gamma$ ) and IL-2 signaling. Thereafter, the intermediate phase of tissue remodeling takes place with ECM remodeling, collagen degradation, muscle and osteoblast differentiation, and cell proliferation and migration accompanied by cytokine signaling. The late stages were less dynamic in terms of pathway enrichments and were associated with proteoglycan and tissue metabolism, attenuation of SMC proliferation, and increase in contractile machinery along with continued chondro-osteogenic differentiation. Similar analysis in uninjured arteries revealed an overall less dynamic response but with apparent changes in the endothelial signaling and response to corticosteroids early after injury to the contralateral artery, followed by increased lipid and glucose metabolism, macrophage-derived foam cell differentiation, and bone mineralization corresponding to intermediate-phase and late-stage ossification processes (Supplementary Table IX, online only). With respect to gene ontology categories based on downregulated genes, less enrichment was noted in general and mostly related to altered neural function and autophagy in both injured and uninjured arteries.

Key transcription factors and network drivers were also predicted on the basis of all significantly dysregulated genes at each time point (Fig 3). Here, an early involvement of Spl and Smad3 transcription factors associated with smooth muscle phenotypic modulation and Tp53 and Brcal related to cell apoptosis or healing was noted. Intermediate phase was marked with involvement of smooth muscle-related Srf, proliferation factor Cdknlb, and stem cell factor Pou5fl (Oct4). Another stem cell transcription factor, Pax3, appeared also in late stage on injury. Neural factors were represented throughout all stages by Notch, Nanog, and Neurodl. When it comes to key network drivers, an early involvement was shown 


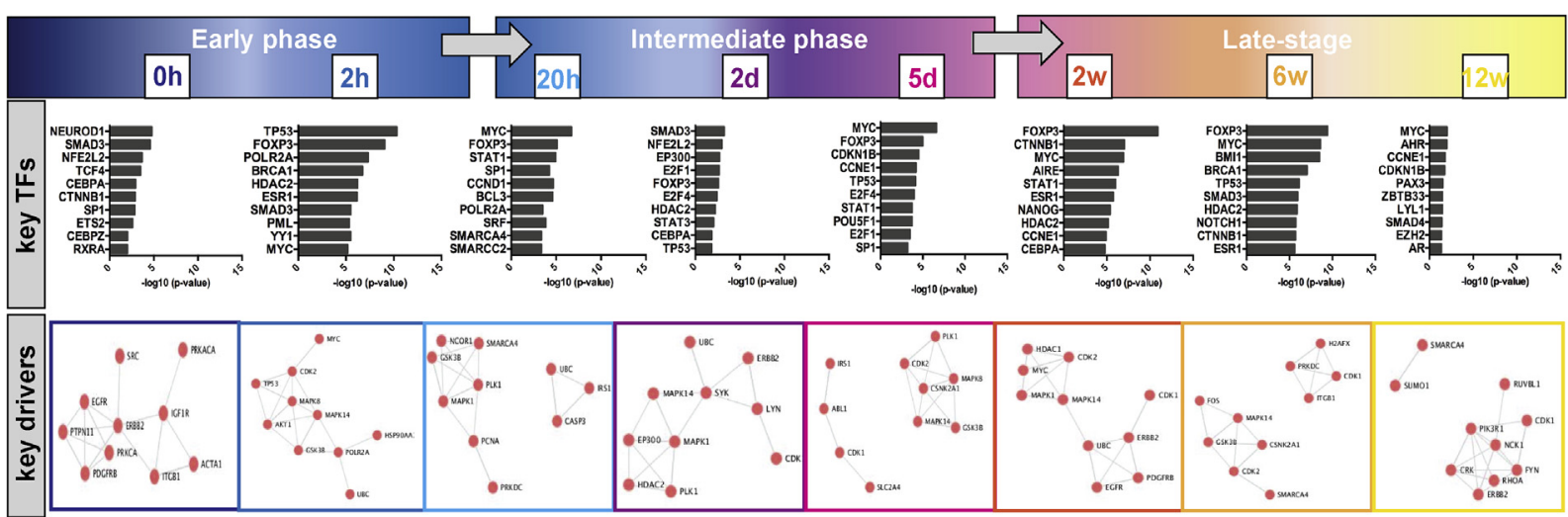

Fig 3. Key drivers and transcription factors (TFS) regulating the gene expression networks in vascular injury. The top 10 most significant key transcription factors predicted to regulate all significantly differentially expressed genes at each time point (both upregulated and downregulated) in injured arteries are presented. Key network drivers were also predicted on the basis of most significant functional coupling and protein interaction nodes among all significantly differentially expressed genes in injured arteries. Closeness of nodes in the network plots signifies similarity in biologic functions. All transcription factors and key drivers predicted with adjusted $P$ value $<.05$.

for Pdgfrb and Igflr in controlling the response to injury, whereas cytoskeletal genes such as Actal and especially Mapk14 were found throughout all stages. General regulators of apoptosis and proliferation Pcna and Casp3 were also found in early stages. In uninjured arteries, the most significant transcription factors throughout the response were circadian rhythm- and metabolismrelated Clock, Tcf4, Ppard, and Ncoal; Igflr again consistently appeared as the key network driver (Supplementary Table X, online only).

The bioinformatic investigation was next extended into the analyses of predicted protein localization (Supplementary Fig 3, online only). Our data show that the biggest changes in protein localization were going on at early time points after injury ( $0-2$ hours), before the period of the highest transcriptional activity. The majority of proteins predicted on the basis of all dysregulated genes during the vascular injury time course were localized to the cell secretome or plasma membrane and a relatively smaller fraction to cytosol, a pattern that was transiently lost during the first 2 to 20 hours of the response and gradually restored later. The same time points coincided with enrichment for mitochondrial and nuclear protein localization, and interestingly, whereas the mitochondrial localization was gradually restored to original low enrichment, the nuclear localization remained at comparably higher level. This analysis indicated that response to vascular injury is dependent on an early shift between secreted, plasma membrane, and cytosolic cellular compartments in favor of mitochondrial and nuclear activity.

Contribution of various cell types during vascular healing. Next, we aimed to link the transcriptomic changes with various cell types present in the vessel wall. First, we assessed expression levels for typical markers of major cells involved in this model (Fig 4, A), showing that there was a major downregulation of classic SMC contractile markers from 20 hours to 5 days after injury, with gradual upregulation thereafter. Their expression profile was inversely related to the expression of replication markers. As expected because of the vessel denudation, endothelial cell markers were repressed early on and up to 5 days after injury, when expression was noted again. Conversely, markers of platelets were upregulated in the acute stages directly after injury and generally subsided from 20 hours after injury. When it comes to immune cells, lymphocyte markers were most prominent at 20 hours to 2 days after injury (some of them were detectable also in the intact artery, such as Fabp4 and Fas), directly after injury (CD3, CD274), and later in the healing stages (Fabp4, Fas, Faslg), whereas others (Itgam, Itgal) were strongly upregulated in the tissue remodeling stages coinciding with the downregulation of SMC markers. Most macrophage markers were also expressed during the intermediate response phase, at 20 hours to 5 days after injury.

These data could be confirmed on the protein level for representative markers by immunohistochemistry of different injury time points (Fig 4, B). For example, the loss of SMC marker Smtn was confirmed during the tissue remodeling stages and regained later, being gradually localized first in the deeper medial layers of the artery and subsequently also in neointimal cells. Lymphocyte presence was assessed using CD8 staining and observed primarily in adventitia of the injured arteries (Fig 4, B, inset), as was the macrophage marker CD68 during tissue remodeling stages (Fig 4, B, inset). Of note, whereas the endothelial cell marker von 
A
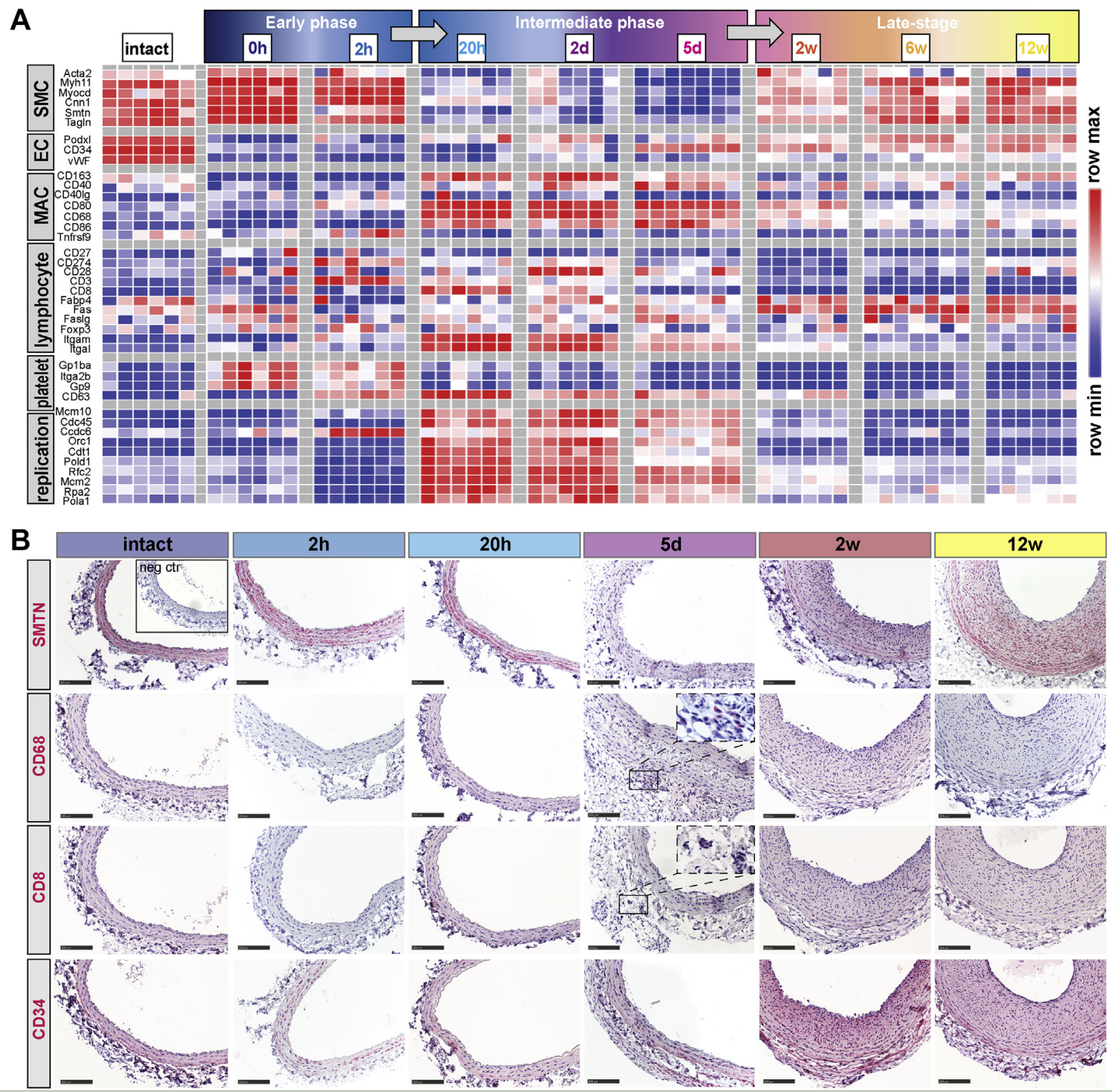

Fig 4. Expression and localization of typical cell markers in vascular injury. A, Heat plot shows messenger RNA expression variations across different time points in injured arteries for classic markers of major cell types and processes related to coagulation and replication. B, Representative typical markers for smooth muscle cells (SMC; Smtn), macrophages (MAC; CD68), lymphocytes (CD8), and endothelial cells (EC; CD34) were assessed on protein level by immunohistochemistry in intact and injured arteries. Scale bar, $100 \mu \mathrm{m}$.

Willebrand factor was completely absent from the tissue (data not shown), CD34 showed a broad expression in medial SMCs during the healing response.

Furthermore, using microarray deconvolution analyses based on expression signatures of various cellular subtypes, ${ }^{18}$ we were able to enumerate the relative cell contributions at each stage during the vascular healing process (Fig 5). As expected, classic endothelial cells were detectable only as a minor fraction in the intact artery and completely absent after injury. The dominant signal after injury was a decrease in the differentiated SMC fraction that was mirrored by an increase in the modulated SMC fraction. During later stages, the differentiated SMC population re-emerged, with a corresponding decrease in the modulated SMC population, indicating redifferentiation of SMCs during the tissue remodeling phases. These results highlight this important cell state transition during the response to injury and validate the use of the deconvolution approach. Also in response to injury, we observed a transient increase in the population of pericytes, whereas fibroblasts showed a burst at 5 days and 12 weeks after injury (Fig 5, A). Neuronal cell fraction already showed a significant decrease from 2 hours of 

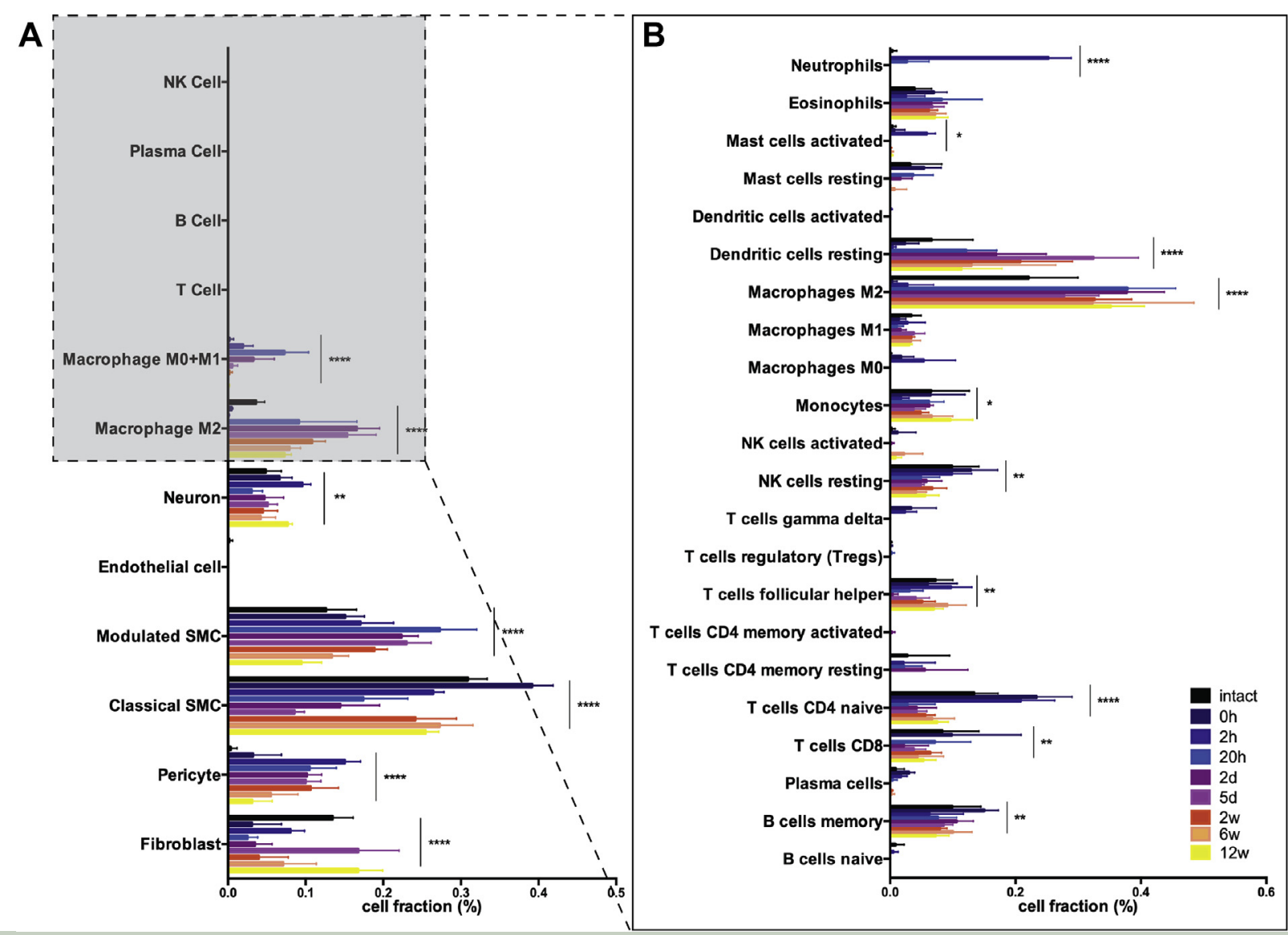

Fig 5. Contribution of various cellular subphenotypes to vascular injury. Bioinformatic deconvolution analysis of bulk microarray profiles was performed for all major cell types from injured arteries, resulting in contribution of relative cell fractions across time points (A). Considering the dominant contribution of smooth muscle cell (SMC) subphenotypes in this analysis and underestimation of immune cell contribution, deconvolution of immune signatures was performed also in more detail separately (B). $P$ values calculated by analysis of variance; ${ }^{*} P<.05$, ${ }^{* *} P<.01,{ }^{* * *} P<.001,{ }^{* * * *} P<.0001$.

injury. This analysis also indicated considerable alterations in the macrophage fractions, but it was suboptimal for assessing all immune cell types, considering that major resident cells were relatively more abundant.

Therefore, we next specifically focused on deconvoluting the immune cell contribution (Fig 5, B, zoomed box), which confirmed a massive signal for neutrophils 2 hours after injury that rapidly subsided thereafter. An early increase in activated mast cells, MO macrophage markers, and naive CD4 and CD8 cells was also indicated during the acute response. At intermediate stages linked with tissue remodeling and SMC proliferation/migration, an increase in resting dendritic fraction and M2 macrophages was dominant. This M2 macrophage-associated cell fraction remained high in signal even at late stages after injury.

Here, using a combination of bioinformatic and histologic tools, we were able to characterize the cellular interplay during the vascular injury and healing response and to elucidate the succession of early immune activation driven by neutrophils, SMC phenotypic modulation in intermediate phases, and late-stage resolution involving M2 macrophages.

Markers of pluripotency, inflammatory response, and osteochondrogenic differentiation are induced in SMCs during response to vascular injury. Because our bioinformatic analyses revealed the involvement of stem cell and pluripotency transcription factors Pou5fl (Oct4) and Pax3, pathways related to osteochondrogenic differentiation, and a contribution of inflammation even at late stages of vascular healing, we next decided to evaluate these novel observations by immunohistochemistry (Fig 6).

Expression of classic osteochondrogenic genes, transcription factor Runx2 and extracellular cartilaginous matrix marker Acan, was gradually induced after injury and peaked at 5 days for Runx 2 followed by Acan at 2 weeks. In correspondence with this, immunohistochemistry showed positive staining for Runx2 and Acan 


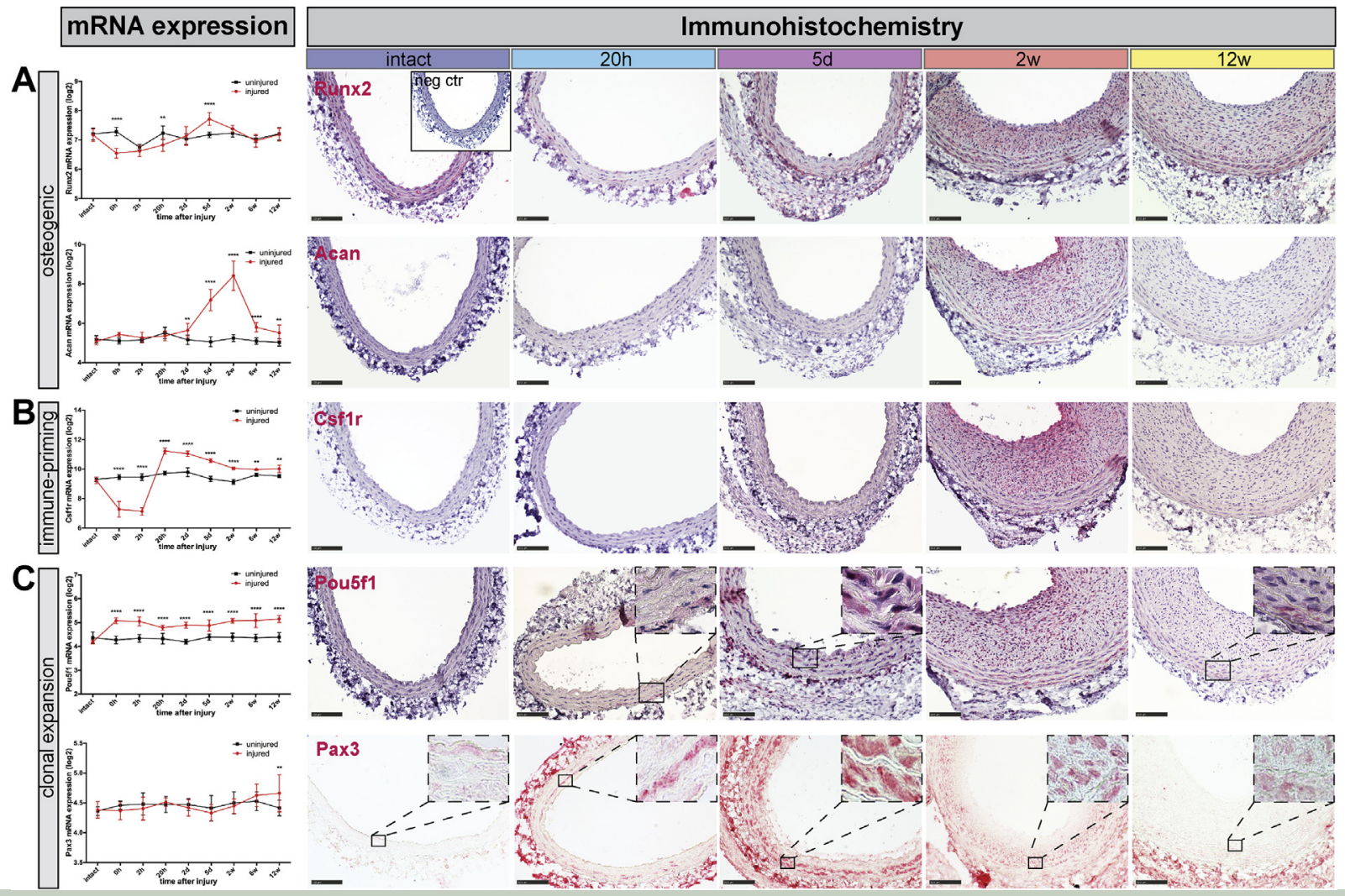

Fig 6. Evaluation of novel findings from analyses of key transcription factors and pathways in response to injury. Messenger RNA ( $m R N A$ ) expression variations and protein immunohistochemical localization across different time points in response to vascular injury are shown for (A) markers of osteochondrogenic differentiation (Runx2 Acan), (B) markers of smooth muscle response to inflammation (Csflr), and (C) pluripotency-related transcription factors (Pou5fl, Pax3). The mRNA expression plots show comparison of transcript levels between injured and uninjured arteries. Histologic images are from intact and injured arteries. $P$ values are calculated at each time point in injured artery compared with uninjured; ${ }^{*} P<.05,{ }^{* *} P<.01,{ }^{* * *} P<.001,{ }^{* * *} P<.0001$. Scale bar, $100 \mu \mathrm{m}$.

proteins within medial and neointimal SMCs and return to baseline levels at 12 weeks after injury (Fig 6, A).

We also found induced transcription of markers typically associated with macrophages (Csflr) from 2 to 20 hours after injury. However, besides immune cells, Csflr was localized to both medial and neointimal SMCs from 5 days after injury, and its expression subsided at later stages (Fig 6, B).

Transcription factor Oct4 showed a small but strongly significant induction in messenger RNA (mRNA) expression directly after injury; immunohistochemistry confirmed that Oct $^{+}$cells were detectable in the vasculogenic zone/adventitia-media border at 20 hours after injury (Fig 6, C). This signal was massively amplified at day 5 and thereafter, when the majority of SMCs in the neointima and underlying media were positive. The signal was attenuated at 6 to 12 weeks after injury with only a few remaining positive cells in the media. Pax3 mRNA expression was increased late at 12 weeks after injury, but $\mathrm{Pax}^{+}$nuclear signal was observable in the medial and neointimal SMCs from 20 hours after injury.
Bioenergy and cholesterol metabolism changes underline systemic response to vascular injury. Because bioinformatic analyses in uninjured arteries indicated an enrichment of pathways related to hormone response and lipid and glucose metabolism, driven by key transcription factors implicated in circadian rhythm such as CLOCK and TCF4, this finally caused us to further assess the systemic metabolic changes in both vascular tissue and plasma during the healing response.

Bioenergy profile bioinformatic analyses ${ }^{19}$ based on microarrays from injured arteries (Fig 7, A) showed rapid alterations in arachidonate and glutathione inositol phosphate metabolism that were acutely induced 2 hours after injury but subsided from 20 hours, to be succeeded by induction of amino and nucleotide sugar metabolism, sphingolipid metabolism, and transfer RNA biosynthesis that were pronounced until 2 weeks af ter injury. Parts of amino acid and glycerophospholipid metabolism and branched amino acid degradation were high during early stages and again later when tissue homeostasis was restored, whereas fatty acid metabolism did not show an obvious pattern in the tissue. 

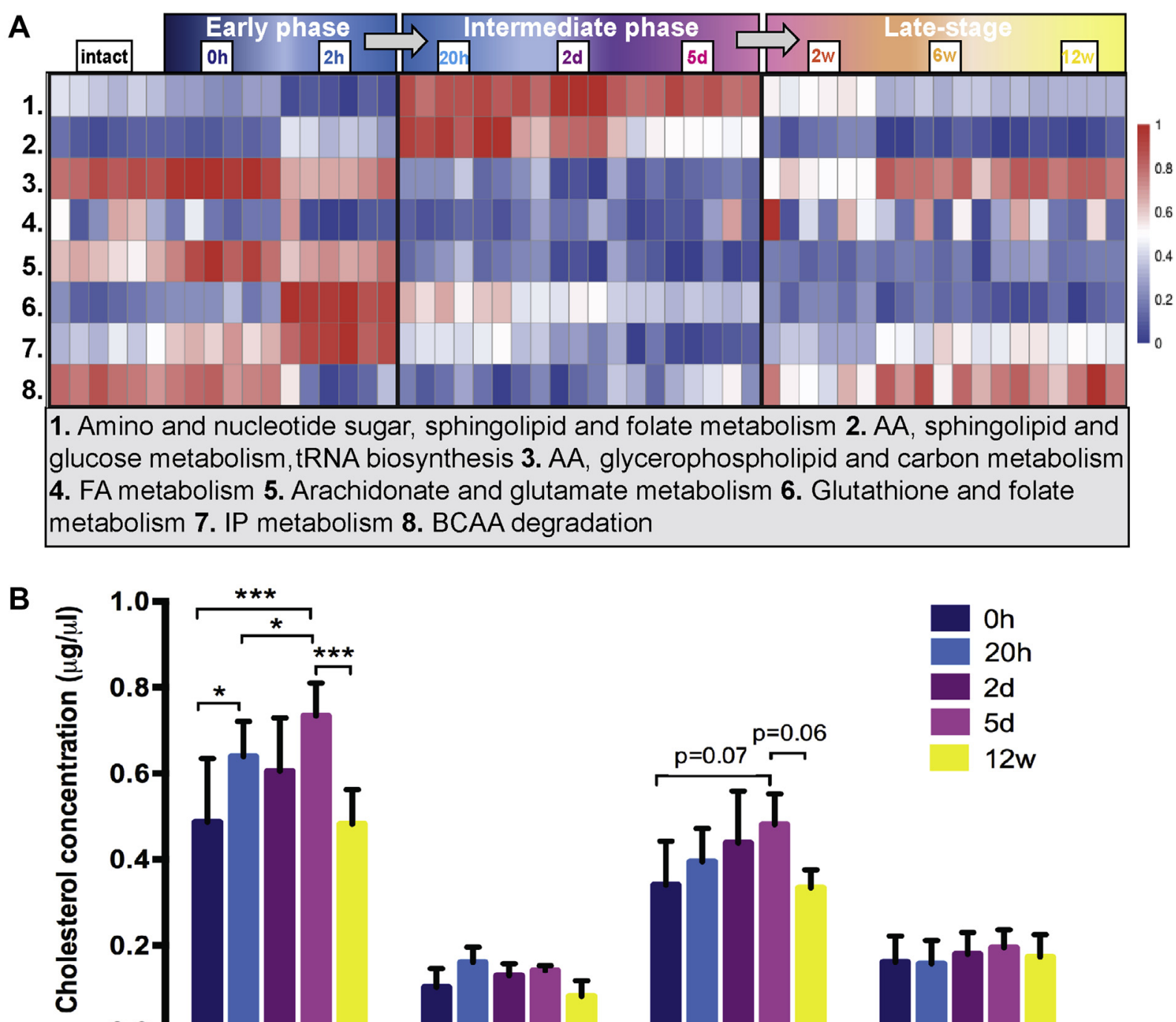

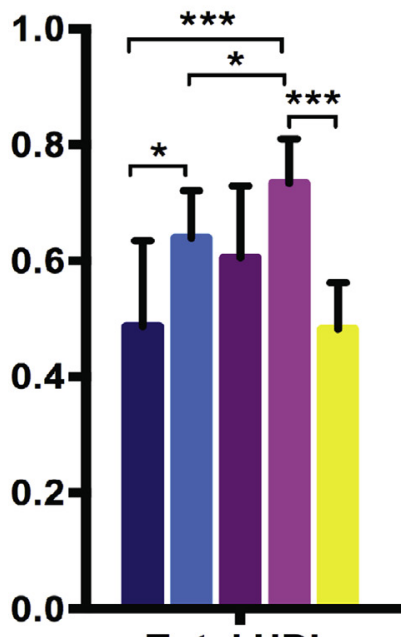

Total HDL

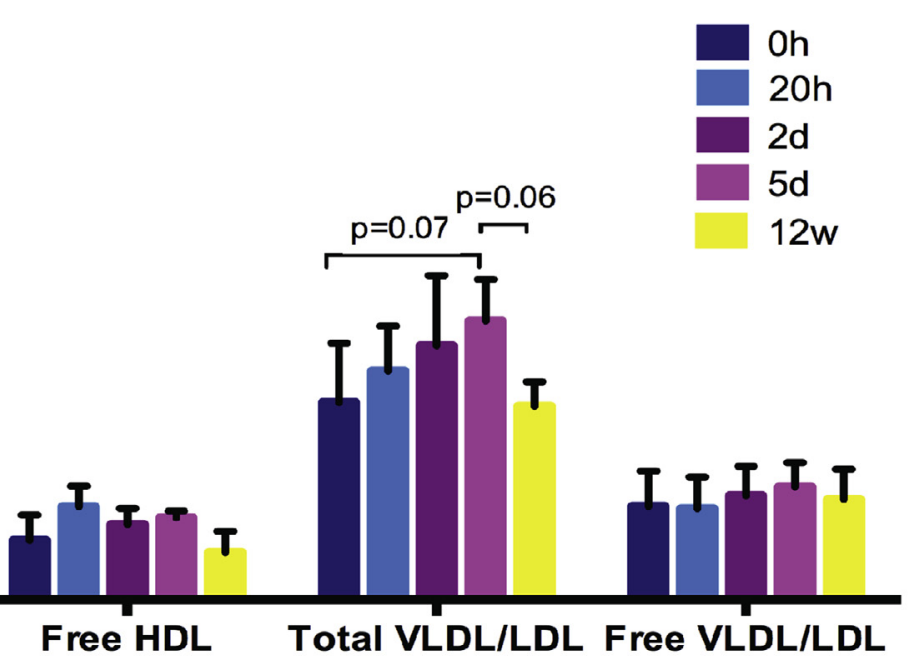

Fig 7. Assessment of the metabolic response to vascular injury in tissue and plasma. Alterations in the bioenergy profile after arterial injury were analyzed using a bioinformatic approach based on microarrays. $\mathbf{A}$, The heat plot shows enrichment of signaling related to eight metabolic modules. AA, Amino acid; BCAA, branched chain amino acid; FA, fatty acid; IP, inositol phosphate; tRNA, transfer RNA; shades of red, high enrichment; shades of blue, low enrichment. B, Plasma cholesterol measurements were performed to profile the levels of total and free cholesterol in the high-density lipoprotein $(H D L)$ and very-low-density lipoprotein/low-density lipoprotein (VLDL/LDL) fractions at different time points after injury. $P$ values calculated by two-way analysis of variance; ${ }^{*} P<.05$, ${ }^{* *} P<.01$, ${ }^{* * *} P<.001,{ }^{* * * *} P<.0001$.

However, systemic cholesterol measurements (Fig 7, B) showed that there was already a significant increase in total plasma high-density lipoprotein levels 20 hours after injury, which normalized back to baseline 12 weeks after injury. Similar trends were also observed for total verylow-density lipoprotein/low-density lipoprotein fraction but did not reach significance.

This analysis confirmed that response to vascular injury is accompanied by profound changes in overall tissue bioenergy metabolism as well as circulatory lipid levels, reaching homeostasis in concert with the transcriptomic expression patterns.

\section{DISCUSSION}

Here, we used an established experimental model of arterial injury in combination with global transcriptomic profiling to create a biobank (BiRCA) and an encyclopedia of the temporal alterations during the arterial wall healing process. Our analyses confirmed the presence of three distinct phases in the healing response: early acute response to injury, intermediate response with tissue remodeling, and late resolution phase. At each phase, we could identify dysregulation of specific genes, pathways, key network drivers, and transcription factors related to the characteristic biologic processes. 
Early response to arterial injury. The earliest time point after injury was characterized by latency in global gene expression changes, observed as a resemblance to intact arteries in the PCA. The acute response after balloon injury has previously been thoroughly investigated and is characterized by endothelial denudation, platelet aggregation, and subsequent recruitment of circulating leukocytes. ${ }^{15,20}$ As expected, our results reveal significant downregulation of endothelial cell-associated genes with a concomitant upregulation of genes associated with leukocytes and inflammation. Upregulation of $\mathrm{Cx} 3 \mathrm{crl}$ that emerged in our analysis has been previously described in leukocyte migration and implicated in SMC differentiation during vessel wall healing. Cleclb has been associated with NK cells and downstream proinflammatory signaling mediated through SMCdependent platelet activation. ${ }^{21}$ Also, the downstream effect of TIr7 has been suggested to influence arterial wall healing because its activation increases nuclear factor $\kappa B$ signaling in SMC proliferation and intimal hyperplasia formation. ${ }^{22}$ An early upregulation of Ednrb was observed, which has previously been shown also to occur in primary SMCs in vitro. ${ }^{23}$ Our results also reveal downregulation in IL-6 expression during the acute phase, which is in concordance with previous studies ${ }^{24,25}$ in which IL- 6 has been associated with tissue metabolism, anti-inflammatory response, and muscle regeneration. $^{26}$ Analysis of related pathways, predicted transcription factors, and key network drivers confirmed the upregulation of coagulation and inflammation in this phase. In addition, these results suggested a simultaneous interplay of cell activation, proliferation, and apoptosis, which was also reflected as a dysregulation of genes related to IL-2 and IFN- $\gamma$ pathways. Upregulation of IL-2 and IFN- $\gamma$ signaling has been associated with Tcell responses and apoptosis of vessel wall resident macrophages through endoplasmic reticulum stress ${ }^{27}$ but also SMC migration and proliferation and intimal hyperplasia. ${ }^{28,29}$ The predicted transcription factors Spl and Smad3 have been implicated in early changes leading to SMC activation in response to platelet-derived growth factor subunit B and transforming growth factor $\beta$ (TGF- $\beta$ ) signaling, respectively, whereas Tp53 and Brcal mediate cell apoptosis and could be related to the mechanical trauma inflicted to the tissue..$^{30,31}$

Intermediate phase with cell dedifferentiation, migration, and structural tissue remodeling. The global gene expression profile in the tissue remodeling phase was marked by distinct dynamics and upregulation of both classic and atypical genes related to ECM, cell migration, and SMC proliferation. For example, upregulation of Tnn can be induced by tumor necrosis factor $\alpha$ and bone morphogenetic protein 2, and the downstream effect is associated with alterations in ECM and cell motility and migration, ${ }^{32}$ whereas Thbs 4 activation results in SMC proliferation and recruitment of macrophages. Also, depletion of Thbs4 has been shown to directly decrease intimal hyperplasia. ${ }^{33}$ Increased expression of Clqtnf3 induced by TGF- $\beta$ signaling has been associated with SMC calcification and Runx2 expression. $^{34}$ Similarly, pathway analysis revealed an upregulation of genes related to ECM modulation, muscle cell proliferation and migration, and cytokine signaling pathways, and we even detected dysregulation of the osteoblast differentiation pathway. Among the transcription factors, SRF was identified, which is a key marker of SMC contractile phenotype and indicates development of processes related to SMC phenotypic modulation $^{35}$ together with the involvement of Cdknlb regulator of proliferation. ${ }^{36}$ The combination of SMC dedifferentiation with simultaneous antiproliferative signaling could relate to the complex interplays necessary to achieve cellular migration and neointimal invasion. Moreover, fundamental interactions between the ECM and SMC surface integrins are necessary to trigger intracellular signaling and cell cycle entry, and ECM interactions also control the availability and activity of growth factors such as heparin-binding mitogens, which can be sequestered by heparan sulfate-containing ECM components to regulate SMC proliferation. ${ }^{37,38}$

Late-phase response and return to vessel wall homeostasis. Late-stage response was characterized by a gradual return to homeostasis and global gene expression profiles similar to the intact arteries by PCA. Analysis of specific gene expressions revealed upregulation of genes related to cytoskeleton, vasodilation, and bone formation and downregulation of genes related to cell proliferation and ECM degradation. For example, Actal was upregulated; it transcribes several isoforms of actin that are fundamental components of cytoskeleton and the contractile apparatus and is a marker for quiescent SMCs. ${ }^{35}$ The downstream effect of Bmp2 is also associated with inhibition of vascular SMC proliferation. ${ }^{39}$ Enrichment analysis revealed upregulation of pathways related to metabolism and SMC contractility with subsequent reduction in cellular proliferation, which also suggested modulation toward the quiescent phenotype. Similar to the tissue remodeling phase, an upregulation in the chondrocyte differentiation pathway was observed. Interestingly, we could detect a significant regulation by transcription factor Tp53 during late homeostasis, which could be explained by its extraapoptotic functions, such as induction of cell cycle arrest, senescence, and DNA repair. ${ }^{40}$

Contribution of major cell types in the arterial healing process. The overall cellular contributions during arterial wall healing were analyzed using microarray deconvolution approaches combined with individual expression levels of classic cell markers and validation by 
immunohistochemistry. As expected, the acute injury phase was marked by the loss of typical endothelial cells, a temporary upregulation of platelet markers, and a rapid infiltration of neutrophils, T cells, and mast cell activation. $^{41,42} \mathrm{Om}$ histologic evaluation, the injured artery also displayed an increase in immune cells, consisting mostly of adventitial $\mathrm{CD}^{+}$and $\mathrm{CD}^{+} 8^{+}$cells. The tissue remodeling phase was characterized by further upregulation of macrophage markers in the media and downregulation of all contractile SMC markers. Particularly, we found an increase in $M 2$ macrophages and resting dendritic cells in this phase, whereas differentiated SMCs were replaced by modulated SMCs, fibroblasts, and pericytes. Interestingly, when it comes to histologic analysis of SMC contractile markers, we could observe a spatial pattern with differences between deeper medial layers and subintimal or neointimal SMCs, which indicates that dedifferentiation of SMCs occurs in a gradual and spatially distributed fashion. We also detected an abundant medial expression of CD34, which is typically regarded as both an endothelial and hematopoietic stem cell marker, whereas its induction in SMCs has previously been related to TGF- $\beta$ signaling. ${ }^{43}$ Late-stage healing was characterized by an increase in contractile SMC markers on the MRNA and protein level and re-emergence of classic SMCs.

Novel pathways related to vessel wall injury and healing. Lineage tracing studies in mice have elucidated that intimal hyperplasia (as well as atherosclerotic plaques) may be formed not only as a result of medial SMC proliferation but also from clonal expansion of restricted cell populations. ${ }^{44}$ In this study, we identified Pou5fl (Oct4) and Pax3, associated predominantly with response of stem cells to wounding, ${ }^{45,46}$ to be among the key transcription factors during chronic and late-stage healing response, respectively. Although expressed by only a few cells in healthy arteries, both markers were significantly upregulated and gradually more abundant also on the protein level on injury, with Pax3 signal present in the media until late stages. We speculate that Pax3 and Oct4 expression in our model could be associated with resident or circulating vascular stem cells undergoing smooth muscle transformation during the medial response to injury; alternatively, expression of these markers could be a feature restricted to unique resident SMCs with a potential for clonal expansion.

Similarly, lineage tracing studies in atherosclerotic plaques have shown that SMCs are capable of expressing markers classically associated with macrophages. ${ }^{47}$ Here, we also found evidence that could support the inflammatory transformation of SMCs in the vascular wall in response to injury, for example, by expressing macrophage markers such as Csflr during the chronic response phase followed by a decrease in the homeostasis phase. Csflr is a target for Runx transcription factors, ${ }^{48}$ upregulation of which was also found in our model, providing a possible mechanism for Csflr upregulation. These results thus imply that the overall increase in macrophage gene expression during vascular healing may result not only from monocyte infiltration as previously described ${ }^{25}$ but also from expression by SMCs.

Interestingly, our comprehensive analysis assigned a specific time point during vascular remodeling to transcription factors and pathways that are usually found in bone or cartilaginous tissues. Most important, Runx2 transcription factor was elevated 5 days after injury, which could explain the induction of ECM-related Acan that peaked 2 weeks after surgery. Runx 2 was initially described as a master regulator of chondrocyte maturation and osteoblast differentiation and a modulator of bone formation ${ }^{49,50}$ but has recently also been connected to arterial stiffening and SMCs phenotypic switch during medial and intimal calcification. ${ }^{51,52}$ In these pathologic processes, increased expression of Runx2 usually leads to release of hydroxyapatite-loaded vesicles and further calcification of the surrounding matrix ${ }^{53}$; however, no such effect has been observed in our model. In our study, its expression returned to normal levels 6 to 12 weeks after injury, which was also accompanied by a decreasing content of proteoglycans and collagens together with increasing elastin formation within the ECM during late time points. These findings suggest that Runx2-related upregulation of proteoglycans might be a directed reaction to maintain vessel function exposed to excessive biomechanical forces, as previously suggested by Hoefer et al. ${ }^{54}$ In particular, Acan is known for withstanding compression in joint cartilage ${ }^{55}$ and might provide additional mechanical protection for SMCs and facilitate reconstitution of contractile properties.

Although based on bulk tissue microarrays, these novel molecular mechanisms could be identified in our multilevel bioinformatic analysis and pave the way for more sophisticated studies of intimal hyperplasia using single-cell technologies and lineage-tracing reporter animals.

Systemic response to vascular injury. The contralateral uninjured artery is commonly used as negative control in the rat carotid balloon model. However, our results show that a systemic response to injury also induces detectable alterations in gene expression even in the uninjured artery. Bioinformatic analysis in uninjured arteries revealed dysregulation of pathways related to tissue metabolism, neural function, and autophagy; most significant transcription factors were related to metabolism and circadian rhythm, whereas Igfir was identified as the key network driver. Injured artery also showed pronounced alterations related to bioenergy, switching to sphingolipid and glucose metabolism in the tissue remodeling phases. Overall, these changes were 
underlined by systemic effects, detected as temporal alterations in the circulatory lipid content after surgical trauma. Thus, our data indicate that the uninjured artery may not represent a control tissue but rather reflect systemic effects in this model.

Limitations. Microarray and histologic analyses in this study were performed on different parts of the artery. Arterial injury with endothelial denudation induces a re-endothelialization process on injury that continues until about 6 weeks, after which it ceases, leaving the middle third of the artery without endothelial coverage. Because presence of an intact endothelium influences proliferation in the vessel wall as well as intimal hyperplasia formation, it is possible that this could have consequences for interpretation of our results. ${ }^{56}$

Furthermore, the use of bulk tissue microarray technology in our study precluded the identification of individual cells, which would be necessary to track the fate of resident and transient cells in the process of vascular healing. These questions are of key interest and should be investigated in the future using single-cell RNA sequencing and reporter animals.

Considering that bioenergy and plasma cholesterol analysis showed profound metabolic changes after injury in our study, this could serve as a basis for a more comprehensive systemic circulation profiling (including cytokines and cell counts) in future studies of this model.

Importantly, the use of the rat arterial response to injury as a model for human restenosis should be considered with caution. Unlike in larger mammals, the carotid artery in the rat has only rare intimal cells. Although platelet adhesion occurs in injured rat arteries, thrombus formation is minimal, whereas it is prominent after vascular injury in rabbits, pigs, and nonhuman primates. ${ }^{4}$ In addition, only male animals were used in this study; therefore, any generalization of our data should be done with caution. The response to injury in these more complex systems should be further investigated; however, our study offers a contemporary insight into the possible mechanisms controlling these processes.

\section{CONCLUSIONS}

In this study, we performed a comprehensive investigation of the temporal alterations in the transcriptomic and cellular landscape after arterial balloon injury. The healing process develops through distinct phases of initial acute injury response, chronic tissue remodeling, and late homeostasis and is characterized by early involvement of lymphocytes and macrophages and a dynamic SMC dedifferentiation with ECM remodeling, finally resolved by regaining of contractile features. Interestingly, our analysis also indicated novel pathways implicated in this process that require further mechanistic investigation, particularly when it comes to the role of clonal expansion and inflammatory and osteochondrogenic transformation in vascular healing Finally, we reveal that the response to surgical trauma is systemic and induces alterations in the gene expression in intact contralateral artery. Our systematic approach has generated a unique biobank resource available to the research community for studies of the arterial wall healing process.

\section{AUTHOR CONTRIBUTIONS}

Conception and design: SR, UR, TS, BS, MD, AH, PE, UH, AR, LM

Analysis and interpretation: SR, UR, TS, BS, JG, AG, AS, RW OB, JO, JL, TQ, AH, PE, UH, AR, LM

Data collection: UR, TS, BS, MD, ML, MK, LM

Writing the article: SR, UR, TS, BS, JG, AG, AS, RW, ML, MK, AR, LM

Critical revision of the article: SR, UR, TS, BS, MD, JG, RW OB, JO, JL, TQ, AH, PE, UH, AR, LM

Final approval of the article: SR, UR, TS, BS, MD, JG, AC,

AS, RW, ML, MK, OB, JO, JL, TQ, AH, PE, UH, AR, LM

Statistical analysis: JG, AG, AS, RW, LM

Obtained funding: AH, PE, UH, AR, LM

Overall responsibility: LM

$\mathrm{SR}$, UR, and TS participated equally and share co-first authorship.

$A R$ and LM participated equally and share senior authorship.

\section{REFERENCES}

1. Allaire E, Clowes AW. Endothelial cell injury in cardiovascular surgery: the intimal hyperplastic response. Ann Thorac Surg 1997;63:582-91.

2. Curcio A, Torella D, Indolfi C. Mechanisms of smooth muscle cell proliferation and endothelial regeneration after vascular injury and stenting. Circ J 2011;75:1287-96.

3. Clowes AW, Clowes MM, Fingerle J, Reidy MA. Regulation of smooth muscle cell growth in injured artery. J Cardiovasc Pharmacol 1989;14(Suppl 6):S12-5.

4. Schwartz SM, deBlois D, O'Brien ER. The intima. Soil for atherosclerosis and restenosis. Circ Res 1995;77:445-65.

5. Versari D, Lerman L, Lerman A. The importance of reendothelialization after arterial injury. Curr Pharm Des 2007;13 $1811-24$.

6. Newby AC, Zaltsman AB. Molecular mechanisms in intimal hyperplasia. J Pathol 2000;190:300-9.

7. Nikkari ST, Järveläinen HT, Wight TN, Ferguson $M$, Clowes AW. Smooth muscle cell expression of extracellular matrix genes after arterial injury. Am J Pathol 1994;144 1348-56.

8. Toma I, McCaffrey TA. Transforming growth factor- $\beta$ and atherosclerosis: interwoven atherogenic and atheroprotective aspects. Cell Tissue Res 2012;347:155-75.

9. Bennett MR, Sinha S, Owens CK. Vascular smooth muscle cells in atherosclerosis. Circ Res 2016;118:692-702.

10. Perisic Matic L, Rykaczewska U, Razuvaev A, Sabater-Lleal M Lengquist $\mathrm{M}$, Miller $\mathrm{CL}$, et al. Phenotypic modulation of smooth muscle cells in atherosclerosis is associated with downregulation of LMODI, SYNPO2, PDLIM7, PLN, and SYNM. Arterioscler Thromb Vasc Biol 2016:36:1947-61.

11. Nurnberg ST, Cheng K, Raiesdana A, Kundu R, Miller CL, Kim JB, et al. Coronary artery disease associated 
transcription factor TCF21 regulates smooth muscle precursor cells that contribute to the fibrous cap. PLoS Genet 2015;11:e1005155.

12. Turner AW, Martinuk A, Silva A, Lau P, Nikpay M, Eriksson P, et al. Functional analysis of a novel genome-wide association study signal in SMAD3 that confers protection from coronary artery disease. Arterioscler Thromb Vasc Biol 2016:36:972-83.

13. Tulis DA. Rat carotid artery balloon injury model. Methods Mol Med 2007;139:1-30.

14. Touchard AG, Schwartz RS. Preclinical restenosis models: challenges and successes. Toxicol Pathol 2006;34:11-8.

15. Clowes AW, Reidy MA, Clowes MM. Mechanisms of stenosis after arterial injury. Lab Invest 1983:49:208-15.

16. Perisic L, Aldi S, Sun Y, Folkersen L, Razuvaev A, Roy J, et al. Gene expression signatures, pathways and networks in carotid atherosclerosis. J Intern Med 2016;279:293-308.

17. Matic LP, Jesus Iglesias $M$, Vesterlund $M$, Lengquist $M$, Hong MG, Saieed S, et al. Novel multiomics profiling of human carotid atherosclerotic plaques and plasma reveals biliverdin reductase $\mathrm{B}$ as a marker of intraplaque hemorrhage. JACC Basic TransI Sci 2018;3:464-80.

18. Wirka RC, Wagh D, Paik DT, Pjanic M, Nguyen T, Miller CL, et al. Atheroprotective roles of smooth muscle cell phenotypic modulation and the TCF21 disease gene as revealed by single-cell analysis. Nat Med 2019;25:1280-9.

19. Sergushichev AA, Loboda AA, Jha AK, Vincent EE, Driggers EM, Jones RG, et al. GAM: a web-service for integrated transcriptional and metabolic network analysis. Nucleic Acids Res 2016;44:W194-200.

20. Tseng CN, Chang YT, Lengquist M, Kronqvist M, Hedin U, Eriksson EE. Platelet adhesion on endothelium early after vein grafting mediates leukocyte recruitment and intimal hyperplasia in a murine model. Thromb Haemost 2015;113: 813-25.

21. Inoue O, Hokamura K, Shirai T, Osada M, Tsukiji N, Hatakeyama K, et al. Vascular smooth muscle cells stimulate platelets and facilitate thrombus formation through platelet CLEC-2: implications in atherothrombosis. PLoS One 2015;10: e0139357.

22. Cercek B, Yamashita M, Dimayuga P, Zhu J, Fishbein MC, Kaul S, et al. Nuclear factor-KB activity and arterial response to balloon injury. Atherosclerosis 1997;131:59-66.

23. Adner M, Cantera L, Ehlert F, Nilsson L, Edvinsson L. Plasticity of contractile endothelin-B receptors in human arteries after organ culture. Br J Pharmacol 1996;119:1159-66.

24. Fedorov A, Kostareva A, Raud J, Roy J, Hedin U, Razuvaev A. Early changes of gene expression profiles in the rat model of arterial injury. J Vasc Interv Radiol 2014;25:789-96.e7.

25. Li JM, Zhang X, Nelson PR, Odgren PR, Nelson JD, Vasiliu C, et al. Temporal evolution of gene expression in rat carotid artery following balloon angioplasty. J Cell Biochem 2007:101:399-410.

26. Pedersen BK, Steensberg A, Fischer C, Keller C, Keller P, Plomgaard $\mathrm{P}$, et al. The metabolic role of IL- 6 produced during exercise: is IL-6 an exercise factor? Proc Nutr Soc 2004:63:263-7.

27. Zhao Q, Zhou D, You H, Lou B, Zhang Y, Tian Y, et al. IFN- $\gamma$ aggravates neointimal hyperplasia by inducing endoplasmic reticulum stress and apoptosis in macrophages by promoting ubiquitin-dependent liver $X$ receptor- $\alpha$ degradation. FASEB J 2017;31:5321-31.

28. Arumugam P, Carroll KL, Berceli SA, Barnhill S, Wrenshall LE. Expression of a functional IL-2 receptor in vascular smooth muscle cells. J Immunol 2019;202:694-703.

29. Tavakoli NN, Harris AK, Sullivan DR, Hambly BD, Bao S. Interferon- $\gamma$ deficiency reduces neointimal formation in a model of endoluminal endothelial injury combined with atherogenic diet. Int J Mol Med 2012;30:545-52.

30. Suwanabol PA, Kent KC, Liu B. TCF- $\beta$ and restenosis revisited: a Smad link. J Surg Res 2011;167:287-97.

31. Deaton RA, Gan Q, Owens GK. Spl-dependent activation of KLF4 is required for PDGF-BB-induced phenotypic modulation of smooth muscle. Am J Physiol Heart Circ Physiol 2009;296:H1O27-37.

32. Scherberich A, Tucker RP, Degen M, Brown-Luedi M, Andres AC, Chiquet-Ehrismann R. Tenascin-W is found in malignant mammary tumors, promotes $\alpha 8$ integrindependent motility and requires p38 ${ }^{\mathrm{MAPK}}$ activity for BMP2 and TNF- $\alpha$ induced expression in vitro. Oncogene 2005;24:1525-32.

33. LV L, Liang $W$, Ye M, Zhang J, Zhang $H$, Xue $G$, et al. Thrombospondin-4 ablation reduces macrophage recruitment in adipose tissue and neointima and suppresses injury-induced restenosis in mice. Atherosclerosis 2016;247: 70-7.

34. Zhou Y, Wang JY, Feng H, Wang C, Li L, Wu D, et al. Overexpression of $\mathrm{Clq} /$ tumor necrosis factor-related protein-3 promotes phosphate-induced vascular smooth muscle cell calcification both in vivo and in vitro. Arterioscler Thromb Vasc Biol 2014;34:1002-10.

35. Owens GK, Kumar MS, Wamhoff BR. Molecular regulation of vascular smooth muscle cell differentiation in development and disease. Physiol Rev 2004:84:767-801.

36. Boucher JM, Harrington A, Rostama B, Lindner V, Liaw L. A receptor-specific function for Notch2 in mediating vascular smooth muscle cell growth arrest through cyclindependent kinase inhibitor 1B. Circ Res 2013;113:975-85.

37. Hedin U, Roy J, Tran PK. Control of smooth muscle cell proliferation in vascular disease. Curr Opin Lipidol 2004;15: 559-65.

38. Tran PK, Tran-Lundmark K, Soininen R, Tryggvason K, Thyberg J, Hedin U. Increased intimal hyperplasia and smooth muscle cell proliferation in transgenic mice with heparan sulfate-deficient perlecan. Circ Res 2004;94:550-8.

39. Nakaoka T, Gonda K, Ogita T, Otawara-Hamamoto $Y$, Okabe F, Kira $Y$, et al. Inhibition of rat vascular smooth muscle proliferation in vitro and in vivo by bone morphogenetic protein-2. J Clin Invest 1997;100:2824-32.

40. Yogosawa S, Yoshida K. Tumor suppressive role for kinases phosphorylating p53 in DNA damage-induced apoptosis. Cancer Sci 2018;109:3376-82.

41. Clowes AW, Clowes MM, Reidy MA. Kinetics of cellular proliferation after arterial injury. III. Endothelial and smooth muscle growth in chronically denuded vessels. Lab Invest 1986;54:295-303.

42. Thyberg J, Blomgren K, Hedin U, Dryjski M. Phenotypic modulation of smooth muscle cells during the formation of neointimal thickenings in the rat carotid artery after balloon injury: an electron-microscopic and stereological study. Cell Tissue Res 1995;281:421-33.

43. Shi X, DiRenzo D, Guo LW, Franco SR, Wang B, Seedial S, et al. TGF- $\beta / S$ mad3 stimulates stem cell/developmental gene expression and vascular smooth muscle cell de-differentiation. PLoS One 2014:9:e93995.

44. Chappell J, Harman JL, Narasimhan VM, Yu H, Foote K, Simons BD, et al. Extensive proliferation of a subset of differentiated, yet plastic, medial vascular smooth muscle cells contributes to neointimal formation in mouse injury and atherosclerosis models. Circ Res 2016;119:1313-23.

45. Hammachi F, Morrison GM, Sharov AA, Livigni A, Narayan S, Papapetrou EP, et al. Transcriptional activation by Oct4 is sufficient for the maintenance and induction of pluripotency. Cell Rep 2012;1:99-109. 
46. Kubic JD, Young KP, Plummer RS, Ludvik AE, Lang D. Pigmentation PAX-ways: the role of Pax3 in melanogenesis, melanocyte stem cell maintenance, and disease. Pigment Cell Melanoma Res 2008;21:627-45.

47. Shankman LS, Gomez D, Cherepanova OA, Salmon M, Alencar GF, Haskins RM, et al. KLF4-dependent phenotypic modulation of smooth muscle cells has a key role in atherosclerotic plaque pathogenesis. Nat Med 2015;21: 628-37.

48. Himes SR, Cronau S, Mulford C, Hume DA. The Runx1 transcription factor controls CSF-7-dependent and -independent growth and survival of macrophages. Oncogene 2005:24:5278-86.

49. Komori T, Yagi H, Nomura S, Yamaguchi A, Sasaki K, Deguchi K, et al. Targeted disruption of Cbfal results in a complete lack of bone formation owing to maturational arrest of osteoblasts. Cell 1997;89:755-64.

50. Otto F, Thornell AP, Crompton T, Denzel A, Gilmour KC, Rosewell IR, et al. Cbfal, a candidate gene for cleidocranial dysplasia syndrome, is essential for osteoblast differentiation and bone development. Cell 1997;89: 765-71.

51. Speer MY, Li X, Hiremath PG, Giachelli CM. Runx2/Cbfal, but not loss of myocardin, is required for smooth muscle cell lineage reprogramming toward osteochondrogenesis. J Cel Biochem 2010;110:935-47.

52. Sun $\mathrm{Y}$, Byon $\mathrm{CH}$, Yuan $\mathrm{K}$, Chen J, Mao X, Heath JM, et al Smooth muscle cell-specific Runx2 deficiency inhibits vascular calcification. Circ Res 2012;111:543-52.

53. Kapustin AN, Chatrou ML, Drozdov I, Zheng Y, Davidson SM, Soong D, et al. Vascular smooth muscle cell calcification is mediated by regulated exosome secretion. Circ Res 2015;116: $1312-23$.

54. Hoefer IE, den Adel B, Daemen MJ. Biomechanical factors as triggers of vascular growth. Cardiovasc Res 2013;99:276-83.

55. Roughley PJ, Mort JS. The role of aggrecan in normal and osteoarthritic cartilage. J Exp Orthop 2014;1:8.

56. Röhl S, Eriksson L, Saxelin R, Lengquist M, Östenson CG Hedin $U$, et al. Noninvasive in vivo assessment of the reendothelialization process using ultrasound biomicroscopy in the rat carotid artery balloon injury model. J Ultrasound Med 2019;38:1723-31.

Submitted Nov 5, 2019; accepted Jan 31, 2020.

Additional material for this article may be found online at www.jvascsurg.org. 